\title{
DOUBLE-WALL COLLIMATOR DESIGN OF THE SNS PROJECT*
}

\author{
N. Simos ${ }^{\dagger}$, H. Ludewig, N. Catalan-Lasheras, S. Crivello \\ Brookhaven National Laboratory, Upton, New York, USA 11973
}

\begin{abstract}
The collimator absorber array of the Spallation Neutron Source (SNS) project is responsible for stopping the 1.0 $\mathrm{GeV}$ protons that are in the halo of the beam. It is estimated that $0.1 \%$ of the $2 \mathrm{MW}$ beam will be intercepted by the adopted collimating scheme implemented at various sections of the beam transport and accumulation. This paper summarizes the conceptual design of the collimator absorber as well as the supporting detailed analysis that were performed and guided the design process. Key requirement in the design process is the need for the collimator beam tube to minimize beam impedance while closely following its beta function. Due to lack of available experimental data, the long-term behavior of irradiated materials in an environment where they interface with coolant flow becomes an issue. Uncertainties in the long-term behavior prompted a special double-wall design that will enable not only beam halo interception but also the efficient transfer of deposited energy both under normal and off-normal conditions to the coolant flow. The thermo-mechanical response of the double wall beam tube and of a particle bed surrounding it are discussed in detail in the paper.
\end{abstract}

\section{INTRODUCTION}

It has been assumed that $0.1 \%$ of the $2 \mathrm{MW}$ beam will be intercepted by the collimating scheme set at the various sections of the beam transport and accumulation. Shown in Figure 1 is the layout of the generic collimator absorber. It consists of the collimator beam tube, a cooled particle bed that surrounds the beam tube and a ringshaped shielding that in turn surrounds the particle bed and the collimator tube. All components are enclosed within the space of a stainless steel vessel. Because of its functional importance, particular emphasis is given on the design and performance of the collimator beam tube that represents the first proton-intercepting element of the absorber scheme. The need for the collimator beam tube to minimize beam impedance while following its beta function and separate the vacuum space from the pressurized flowing light water environment makes its design challenging. Because of lack of experimental data, the long-term behavior of irradiated material that is wetted on the one surface becomes an issue. Such uncertainties prompted a special double-wall design that

\footnotetext{
* This work was performed under the auspices of the US DOE

† simos@bnl.gov
}

will enable not only beam halo interception but also the efficient transfer of deposited energy to coolant flow.

Under normal operating conditions the collimator will intercept $0.1 \%$ of the beam. The $2 \mathrm{MW}$ proton beam has a frequency of $60 \mathrm{~Hz}$ and pulse duration of $1 \mu \mathrm{s}$. In the RTBT line the proton beam bunches have attained peak energies of $1.0 \mathrm{GeV}$. In the event of beam straying from the designed orbit the full proton beam rather than its halo will penetrate the beam tube and be intercepted by the particle bed. It has been conservatively assumed that two pulses will deposit energy in the bed before the beam is tripped. If such a scenario is anticipated to occur frequently then the integrity of the particle bed or whatever material in its place need to be assessed since it will resemble a target for a couple of pulses.

Given the location and the function of the beam tube within the collimating assembly, a material that exhibits good mechanical strength properties and has good resistance to radiation damage must be selected. The requirements become more stringent due to the fact that there is to be coolant (light water) on the back face of the beam tube. A material that has the required strength and is accompanied by irradiation experience data is Inconel718. Since experience data only involve material free of welds, while in order to satisfy beam impedance requirements a complex transition in the beam tube is introduced that inevitably will contain welds, a double wall concept for the beam tube was introduced. Accordingly, a double Inconel-718 wall separated by a gap filled with pressurized helium has been designed that eases the requirements of no welds in the critical transition region. The purpose of the helium is twofold. On one hand it provides a heat transfer path to the outer wall and on the other hand can be detected in the beam tube vacuum space if a leak in the inner wall occurs. The double wall concept also allows for the separation of the critical inner wall from the light cooling water. In order to help the heat transfer path from the inner wall to the flowing coolant, a cooper wire is introduced within the helium gap. The various elements of the analyses including optimization of the heat transfer path are discussed in the next sections.

\section{ENGINEERING ANALYSIS}

Following the conceptual design of the collimator absorber, thermal and stress analysis of the key elements guided the final absorber configuration. Specifically, 
based on results of energy deposition generated by the neutronic codes and MCNPX [5] under both normal and off-normal conditions, the thermal response of the beam tube was calculated through a detailed finite element analysis coupled with estimates of heat transfer capacity to the coolant flow.

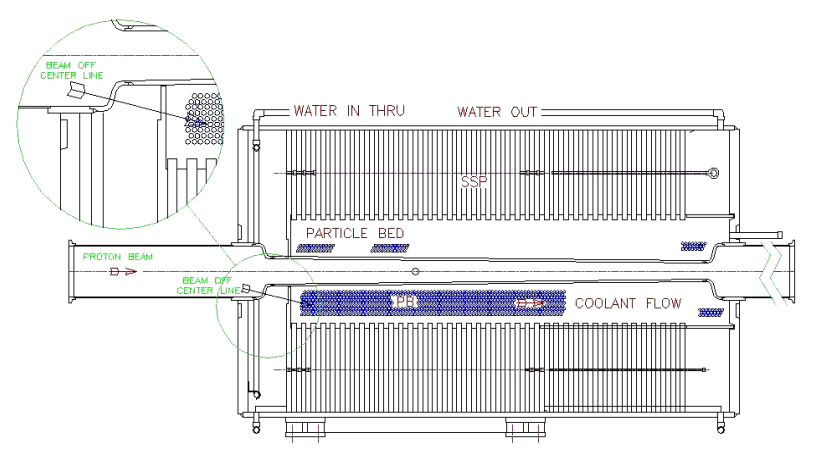

Figure 1. Schematic of the overall collimator/absorber assembly

Following optimization of the thermal problem under normal conditions, the thermal stress response and thermal fatigue problems were addressed using the ANSYS [6] code.

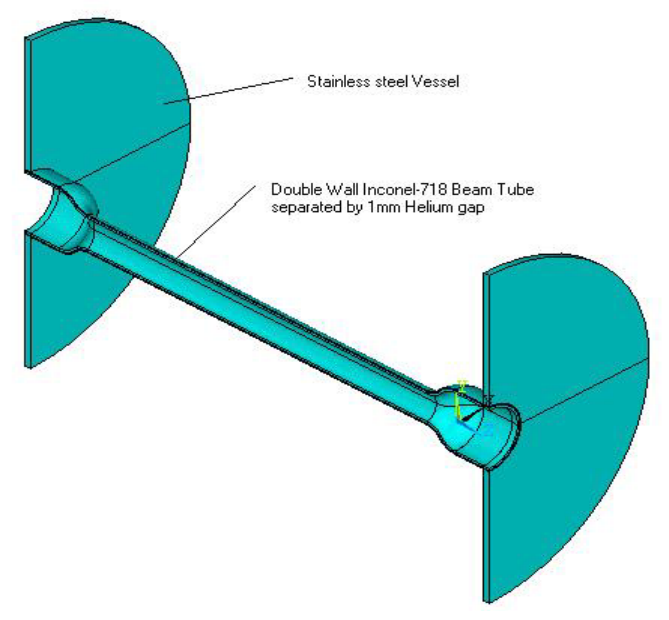

Figure 2. Collimator section used in the thermal and stress analysis using finite elements

\subsection{Thermal Analysis}

The heat deposition rate for normal conditions is determined assuming a parallel beam of $1 \mathrm{GeV}$ protons. The actual beam for such case is ignored in this calculation (not intercepted by the beam tube) and only the halo portion is considered. Radially the halo is assumed to be $3 \mathrm{~mm}$. thick and drops off in a step-wise fashion, with $75 \%$ in the first millimeter, $20 \%$ in the next millimeter, and $5 \%$ in the last millimeter. The fraction of the primary beam assumed to be in the halo is 0.001 , and thus the maximum number of protons in the halo per pulse is $1.0 \times 10^{14}$. Due to uncertainties in both the neutronic calculations and the fact that proton beam will be moving around as a result of instabilities, a $1 \%$ beam interception has been assumed in the calculations of operating temperatures and stresses.

In the critical section of the double wall beam tube (front transition) where the beam halo is first intercepted, the energy deposited and the subsequent temperature rise experienced by the wall material is maximum. While the entire back surface of the outer wall is wetted by the 50 psi coolant flow as it makes its way though the particle bed, the transition section still operates at much higher temperatures. In an accident condition where the $\mathrm{He}$ in the gap has escaped the temperatures could rise dramatically. To circumvent such condition, the heat transfer capacity around the critical section was enhanced. It was achieved by introducing a narrow channel flow that follows the shape of the beam tube transition and increases the velocity of the flowing coolant.

The optimization loop consists of the following steps:

$\S$ Selection of the size of the introduced annular space and the calculation of the velocity of flow $\mathrm{U}_{\mathrm{m}}$ that can be achieved based on the mass flow rate of coolant

$\S$ Estimation of the Reynolds number $\mathrm{RE}_{\mathrm{DE}}=\mathrm{U}_{\mathrm{m}}$ $\mathrm{D}_{\mathrm{E}} / v\left(\mathrm{D}_{\mathrm{E}}\right.$ is the equivalent diameter of the annulus and $v$ is the dynamic viscosity)

$\$$ Estimation of the Nusselt number

$\S \quad \mathrm{N}_{\mathrm{NU}}=0.023\left(\mathrm{RE}_{\mathrm{DE}}\right)^{0.8}\left(\mathrm{~N}_{\mathrm{PR}}\right)^{0.3}$ where $\mathrm{N}_{\mathrm{PR}}=5.85$ for water

$\S \quad$ Calculation of heat transfer film coefficient $\mathbf{h}_{\mathbf{f}}=$ $\mathbf{N}_{\mathbf{N U}} \kappa / \mathbf{D}_{\mathbf{E}}$ where $\kappa$ is the conductivity of the coolant

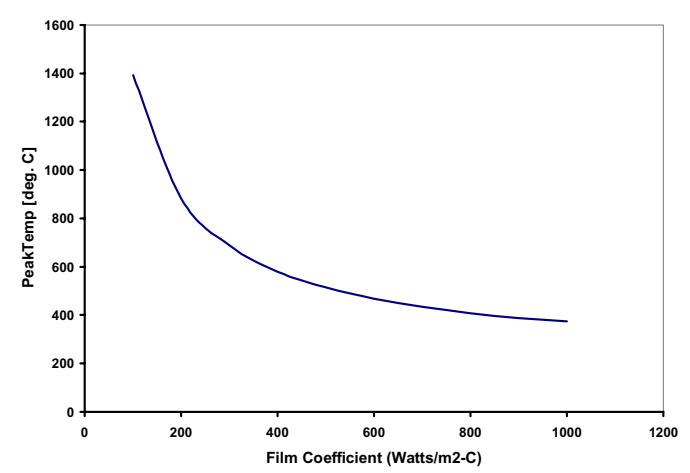

Figure 3. Peak operating temperature as a function of the heat transfer coefficient on the outer inconel wall

A number of different scenarios were thermally analyzed using the energy depositions calculated by the neutronic codes in order to minimize the operating temperatures. Further, attention was paid to accident conditions that may arise, such as loss of helium in the gap, and the consequences were addressed. Table 1 lists the peak temperatures for different combinations of contributing factors including radiation heat transfer between the surfaces in the helium gap. It is apparent from Table 1 that the copper wire dominates the heat transfer path. Even in an accident condition, the operating temperature will be below critical values for the material. 
Table 1. Peak temperatures $\left({ }^{\circ} \mathrm{C}\right)$ in beam tube

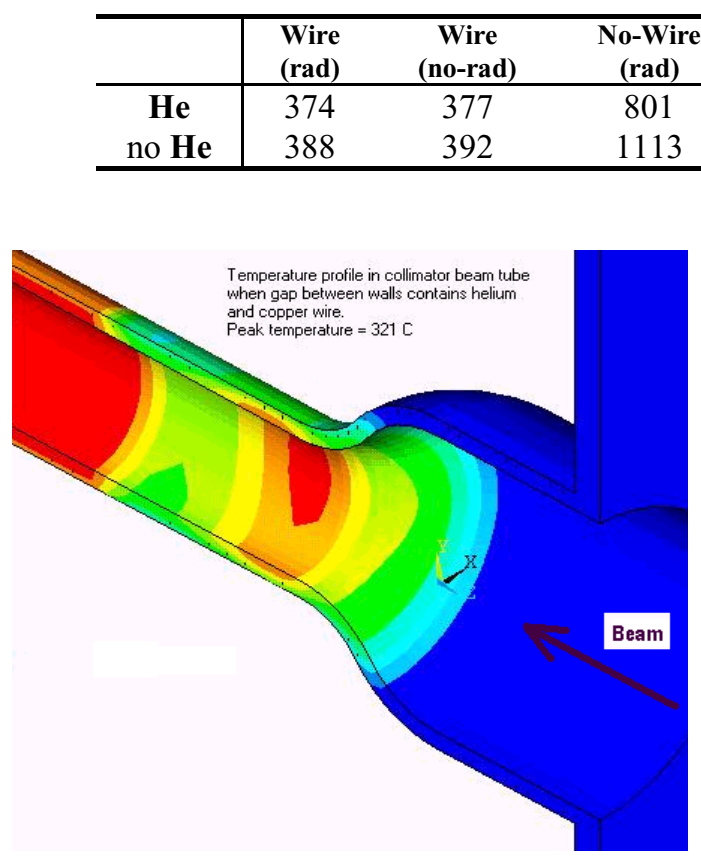

Figure 4. Temperature distribution in the critical section of the beam tube under normal operating conditions

\subsection{Thermal Stress and Shock Estimation}

Following the thermal analysis of the double wall beam tube, steady state and transient stress analyses were performed. Under the "equivalent" steady condition the thermal operating stresses in the beam tube were calculated as a result of the temperature profile that the system stabilized at under normal operations. Shown in Fig. 5 is the axial stress distribution. With peak stresses around $100 \mathrm{MPa}$, a large safety margin is available. These stresses, however, are to be superimposed onto the stresses generated by the combined effect of pressurized helium in the gap and vacuum inside the beam tube as well as loading from the particle bed onto the beam tube. The combination of all loadings still allows for ample margins on the inconel-718 material that exhibits yield strength of over $1000 \mathrm{MPa}$. The accident scenario of the beam straying into the collimator is shown in Fig. 6. Two full mis-directed pulses will be allowed before the beam is tripped. The induced shock stresses in the double wall, under such conditions, are still low to be of concern.

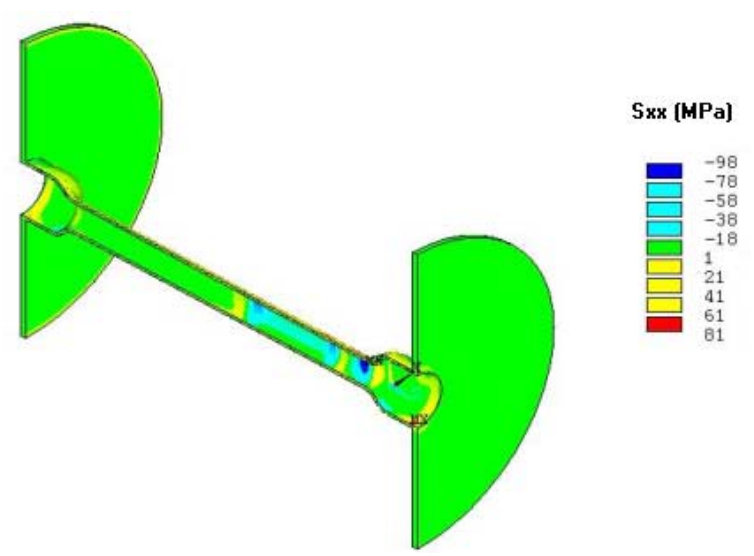

Figure 5. Axial thermal stress for normal conditions

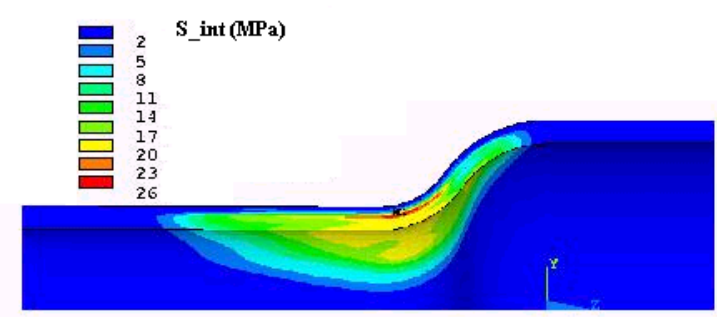

Figure 6. Shock stress generated in the beam tube after full beam strayed into the wall

\section{REFERENCES}

[ 1 ] N. Catalan-Lasheras et al., "Beam Loss and Collimationat SNS Ring," $7^{\text {th }}$ ICFA Mini-Workshop on High Intensity High Brightness Hadron Beams; Wisconsin, September 13-15, 1999

[2 ]H. Ludewig et al., "Collimator Systems for the SNS Ring," PAC 1999, New York, 1999

[ 3 ] N. Catalan-Lasheras et al., "Optimization of the Collimator System for the SNS Accumulator Ring," Physical Review Special Topics - Accelerators and Beams, Vol.3, 2000

[ 4 ] A.J. Chapman, "Heat Transfer," $3^{\text {rd }}$ Edition, McMillan, 1974

[ 5 ] MCNPX Users Manual - Version 2.1.5, L.S. Waters, ed., LANL, Los Alamos, TPO-E83-G-UG-X-00001, 1999

[ 6 ] ANSYS Engineering Analysis of Systems, Swanson Analysis Systems Inc., 1999 